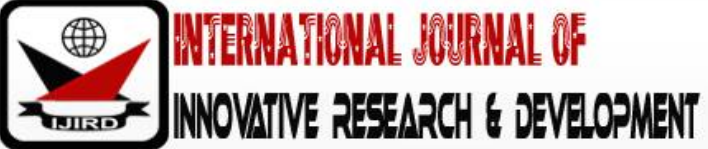

ISSN 2278 - 0211 (Online)

\section{Formulation of Demulsifying Agent for Water in Oil Emulsion Treatment}

\begin{tabular}{|c|}
\hline \hline Paschal Amajuonyi \\
Student, Department of Petroleum Engineering, Federal University of Technology, Nigeria \\
Patrick C. Azubuike \\
Chidudent, Department of Petroleum Engineering, Federal University of Technology, Nigeria \\
Chidiebere C. Omaka \\
Ntudent, Department of Petroleum Engineering, Federal University of Technology, Nigeria \\
Nzenwa Dan Enyioko \\
Angineering, Federal University of Technology, Nigeria \\
Anthony O. Chikwe \\
Lecturer, Department of Petroleum Engineering, Federal University of Technology, Nigeria
\end{tabular}

\begin{abstract}
The production of crude oil along with water has been a primary problem in the petroleum industry. In this work, an emulsion breaking chemical (LFD) was formulated from locally sourced materials and experimentally compared with an imported commercially available demulsifier (sulphuric acid) to determine its effectiveness in crude emulsion resolution. Materials used are Castor oil, locally made liquid soap, Camphor, Alum, Cassava Starch and distilled water. It was tested on a crude oil emulsion sample from the Niger Delta field at a temperature of 850f, under the same experimental condition with the imported commercially available demulsifier (sulphuric acid) as the control. The percentage volume of water separated by the locally formulated demulsifier (LFD) was $93.33 \%$ while the separated percentage volume of water by commercially available demulsifier (sulphuric acid) was $69.33 \%$. This clearly showed that the locally formulated demulsifier had a better performance in terms of emulsion resolution than the commercially available imported demulsifier (sulphuric acid).
\end{abstract}

Keywords: Demulsifiers, castor oil, sulphuric acid, liquid soap, camphor, emulsion, alum, cassava starch, sediment

\section{Introduction}

When two liquids that are immiscible for example oil and water come together in contact, they do not mix homogenously or dissolve to form a solution, instead one liquid gets dispersed in the other liquid. The dispersion usually occurs in aqueous medium to result in an emulsion. The water present in crude oil can bring about undesirable consequences like: Leaching of additives, corrosion, raise conductivity, etc. (Udonne, 2012). For financial and operational reasons, it is important to isolate the water totally from the crude oils before moving them (from one place to another) or refining them. To diminish the water contained in the crude oil produced, the water/ crude oil emulsions must be broken (demulsified). Accordingly, crude oil is a mind-boggling liquid including colloidal particles, for example for example asphaltenes and resins dispersed, it is important to comprehend the mechanisms in charge of the stabilization of these emulsions. (Mohammedetal.,1993).

This crude oil contains basic sediment and water (BS \&W) ranging from 2 to $11 \%$ which is greater than the specified BS \&W (lower than 0.5\%). Therefore, it has somewhat been sold as emulsions at low cost because of the high expense for treating emulsions. Water-in-oil emulsions are created during the production of crude oil which is regularly accompanied with water. The emulsion stability ranges from a couple of minutes to years contingent on the inherent characteristics of the crude oil and to some extent, the inherent characteristics of water. (BhardwajandHartland,1988). So as to reduce the minimum the production problems associated with crude oil emulsions and environmental concerns, petroleum operators need to deter their formation or to break them. At times, when the development of emulsions is an after-effect of poor operation practices, it is possible to deter the formation of emulsion. Nonetheless, in many cases, emulsion formation can be predicted. The exclusion of water during recovery from the oil wells and prevention of agitation is hard to accomplish, and emulsions must be treated. The treatment of water-in-crude oil emulsion include the use of thermal, electrical, chemical process or their blends (Ojinnaka, 2016). 


\section{Materials and Methods}

\subsection{Materials}

The sample of crude oil emulsion utilized in this research was gotten from an oil field in Niger Delta region of Nigeria. Two Demulsifiers, locally formulated demulsifier (LFD) and a commercially available imported $\left(\mathrm{H}_{2} \mathrm{SO}_{4}\right)$ were used in treating this emulsion. The centrifugal agitation method was used to determine the Demulsifier most effective in breaking the emulsion. The materials used in formulating the demulsifier are shown in Table 1 below.

\begin{tabular}{|c|c|c|}
\hline Content & Function & Weight/Vol \\
\hline Alum & To facilitate settling of sediments. & $5 \mathrm{~g}$ \\
\hline Castor Oil & It acts as the lipophilic agent in the demulsifier. & $30 \mathrm{ml}$ \\
\hline Starch & Coalescing of the tiny water droplets. & $5 \mathrm{~g}$ \\
\hline Liquid Soap & Destabilization of emulsion film & $50 \mathrm{ml}$ \\
\hline Camphor & It improves the demulsifier performance & $10 \mathrm{ml}$ \\
\hline
\end{tabular}

Table 1: Materials Used for Locally Formulated Demulsifier (LFD)

Apparatus for the experiment include weighing balance, measuring cylinder (50ml graduated), beaker, magnetic heatstirring machine, filter paper, measuring cylinder (200ml graduated), Hamilton beach mixer, stop watch, syringe, micropipette, thermometer, centrifuge.

\subsection{Methods}

Water-in-oil emulsion was formed in the laboratory as follows:

- $180 \mathrm{ml}$ of crude oil was blended together with $(20 \mathrm{ml})$ of liquid surfactant in a beaker for emulsification. The crude oil/ surfactant blend was placed in a Hamilton beach mixer for agitation.

- Water of $20 \mathrm{ml}$ representing $10 \%$ of the emulsion was then gradually added to the oil phase at a consistent rate of agitation. Agitation continued until the emulsion reached ambient temperature without forced cooling.

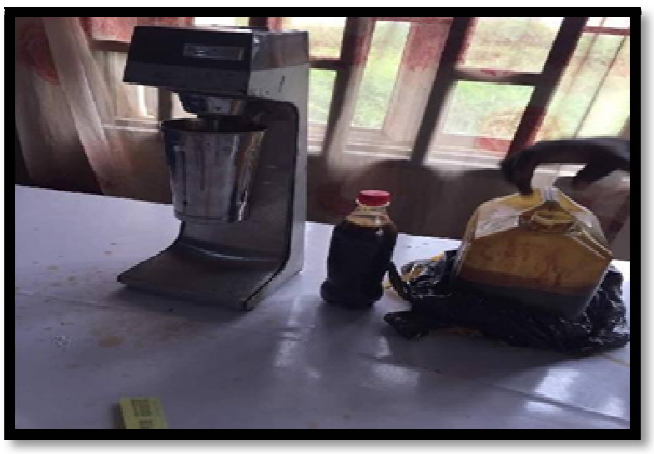

Figure 1: Hamilton Beach Mixer

Locally formulated demulsifier (LFD) procedure are as follows:

- A solution of $10 \mathrm{~g}$ of camphor dissolved in $30 \mathrm{ml}$ of castor oil was stirred and heated $\left(150^{\circ} \mathrm{F}\right)$ in a Magnetic heating and stirring machine until homogeneity of solution is achieved.

- A mixture of $5 \mathrm{~g}$ of starch (from cassava flour), $50 \mathrm{ml}$ of detergent, and $5 \mathrm{ml}$ of Alum solution was prepared in another beaker and put into the camphor castor oil solution.

- All precipitates or sediments were filtered off after the new mix or blend above has been further stirred and healed for 2 hours.

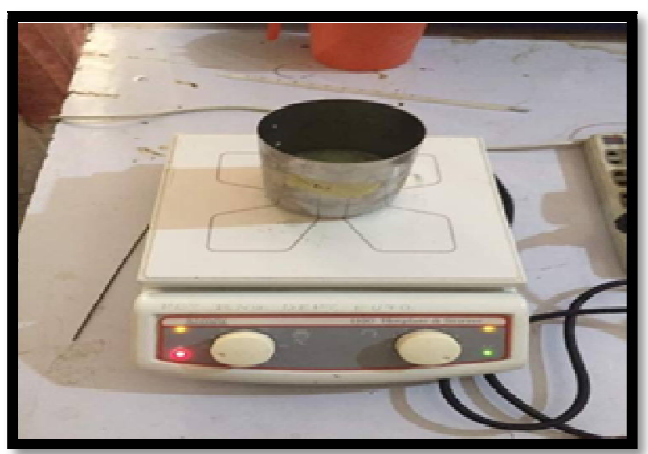

Figure 2: Magnetic Heat-Stirring Machine 


\subsubsection{Analytical Procedure}

The first experiment was done taking sulphuric acid (H2SO4) to be its demulsifying agent while the second was carried out using locally formulated demulsifier (LFD).

- A $75 \mathrm{ml}$ of emulsified crude oil were poured into each of the 12 measuring tubes. All measuring tubes were labeled according to the concentration of Demulsifier to be used for the analysis. Six bottles of demulsifier were utilized with concentrations of 0.2 milli litre, 0.4 milli litre, 0.6 milli litre, 0.8 milli litre, 1.0 milli litre, and 1.2 milli litre of demulsifier.

- All the cylinders were manually agitated properly to ensure fluid mobility

- All tubes containing samples with varying dose of demulsifier were placed into a centrifuge for agitation to achieve proper mixture.

- The revolutions in one minute (rpm) were noted down.

- After the time of rotation had passed, the test tubes placed in the centrifuge were measured and also the separation of the oil from the water recorded

- The procedure was done again for the entire samples and the results were also recorded.

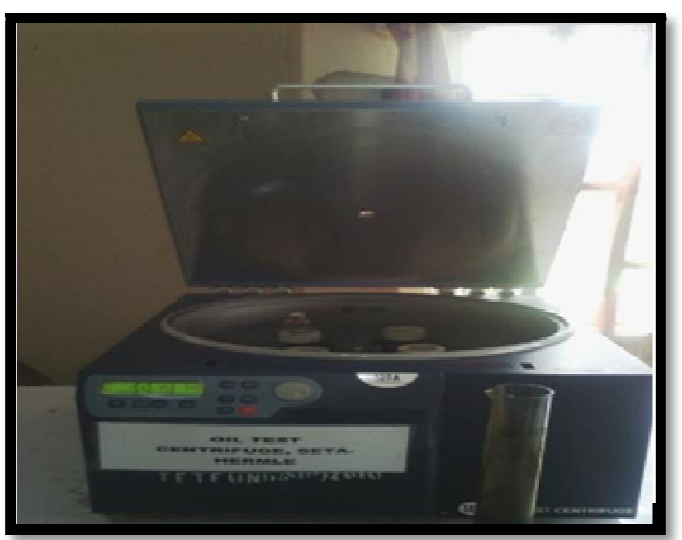

Figure 3: Centrifuge

\section{Results and Discussion}

The results of the emulsion resolution using the two demulsifiers (a locally formulated Demulsifier (LFD) and commercially available Demulsifier (H2SO4)) at a constant treatment temperature of $850 \mathrm{~F}$ and time interval of 2 to 12minutes and at a varying RPM of 1000 and 1500 summarized in Table 2,3.1,3.2,3.3,3.4 and 3.5 accordingly.

\begin{tabular}{|c|c|}
\hline Crude Properties & \\
\hline Density & $0.864 \mathrm{~g} / \mathrm{Cm}^{3}$ \\
\hline Api & $32.46^{\circ}$ \\
\hline Viscosity & $2.9 \mathrm{Cp}$ \\
\hline
\end{tabular}

Table 2: Characteristics of Crude Oil Utilized

\begin{tabular}{|c|c|c|c|c|c|c|}
\hline \multirow{2}{*}{$\begin{array}{c}\text { Vol. of } \\
\text { Demulsifiers(ml) }\end{array}$} & \multicolumn{5}{|c|}{ Separated Water Volume(ml) } \\
\cline { 2 - 7 } & $2 \mathrm{~min}$. & $4 \mathrm{~min}$. & $6 \mathrm{~min}$. & $8 \mathrm{~min}$. & $10 \mathrm{~min}$. & $12 \mathrm{~min}$. \\
\hline 0 & 0 & 0 & 0 & 0 & 0 & 0 \\
\hline
\end{tabular}

Table 3: Emulsion without Demulsifier at a Speed of 1000 Rpm

\begin{tabular}{|c|c|c|c|c|c|c|}
\hline \multirow{2}{*}{$\begin{array}{c}\text { Vol. of } \\
\text { Demulsifier(M) }\end{array}$} & \multicolumn{6}{|c|}{$\begin{array}{c}\text { Separated Water } \\
\text { Volume (MI) }\end{array}$} \\
\cline { 2 - 7 } & 2 min. & 4 min. & 6 min. & 8 min. & $10 \mathrm{~min}$. & $12 \mathrm{~min}$. \\
\hline 0.2 & 0.5 & 0.7 & 0.8 & 1.0 & 1.1 & 1.2 \\
\hline 0.4 & 0.9 & 1.1 & 1.3 & 1.5 & 2.0 & 2.2 \\
\hline 0.6 & 2.2 & 2.5 & 2.5 & 2.7 & 2.8 & 2.8 \\
\hline 0.8 & 2.9 & 3.0 & 3.2 & 3.2 & 3.2 & 3.3 \\
\hline 1.0 & 3.0 & 3.1 & 3.3 & 3.5 & 3.5 & 3.6 \\
\hline 1.2 & 3.6 & 3.7 & 3.9 & 4.0 & 4.0 & 4.1 \\
\hline
\end{tabular}

Table 4: Emulsion Treatment with Sulfuric Acid at 1000 Rpm and 85 F

Percentage volume of water separated using $\mathrm{H}_{2} \mathrm{SO}_{4}$ at $1000 \mathrm{rpm}$

$=$ Vol. water separated $\quad$ x 100

Total vol. of water in the emulsion 1 
$=\underline{4.1} \times \underline{100}$

$7.51=54.67 \%$.

\begin{tabular}{|c|c|c|c|c|c|c|}
\hline \multirow{2}{*}{$\begin{array}{c}\text { Vol. of } \\
\text { Demulsifier(ml) }\end{array}$} & & \multicolumn{4}{|c|}{ Separated Water Volume (ml) } & \multirow[b]{2}{*}{$12 \mathrm{~min}}$. \\
\hline & $2 \mathrm{~min}$. & 4 min. & $6 \mathrm{~min}$. & $8 \mathrm{~min}$. & $10 \mathrm{~min}$. & \\
\hline 0.2 & 0.6 & 0.9 & 1.3 & 1.5 & 1.6 & 1.8 \\
\hline 0.4 & 1.0 & 1.4 & 1.5 & 1.8 & 2.2 & 2.4 \\
\hline 0.6 & 2.3 & 2.6 & 2.9 & 2.9 & 3.1 & 3.2 \\
\hline 0.8 & 3.0 & 3.3 & 3.6 & 3.8 & 3.9 & 3.9 \\
\hline 1.0 & 3.9 & 4.3 & 4.9 & 5.1 & 5.1 & 5.3 \\
\hline 1.2 & 4.2 & 4.8 & 4.9 & 5.2 & 5.3 & 5.5 \\
\hline
\end{tabular}

Table 5: Emulsion Treatment with LFD at 1000 Rpm and 85ㅇ

Percentage volume of water separated using LFD at $1000 \mathrm{rpm}$.

$=$ Vol. water separated 100 $* 100$

Total vol. of water in the emulsion 1

$\underline{5.5} \times 100$

$7.5 \quad \frac{5.5}{1}=73.33 \%$

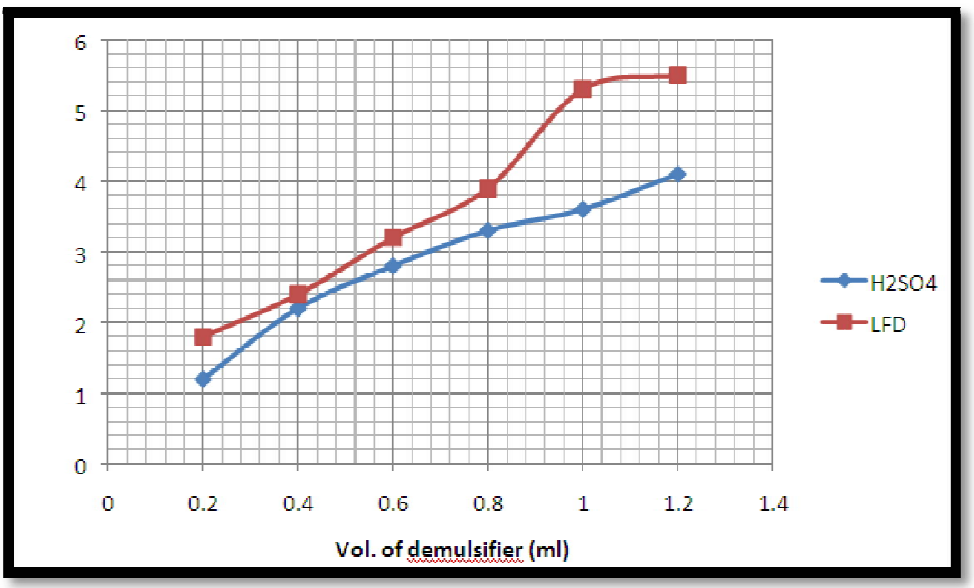

Figure 4: Volume of Separated Water Vs Volume of Demulsifier (Ml) at 85of and 1000 Rpm.

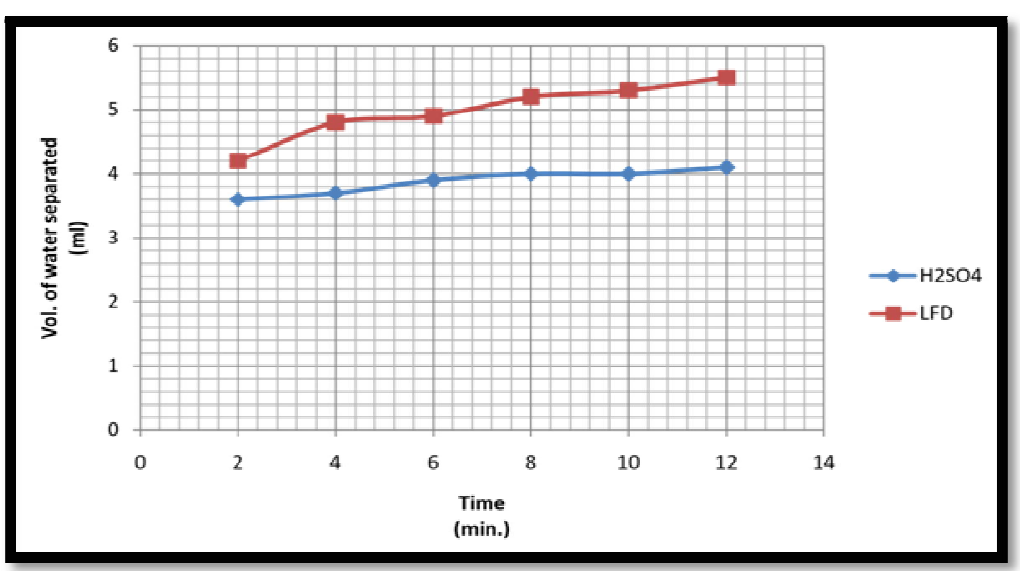

Figure 5: Volume of Separated Water (M) Vs

Time (Min.)@1000 Rpm and 85of 


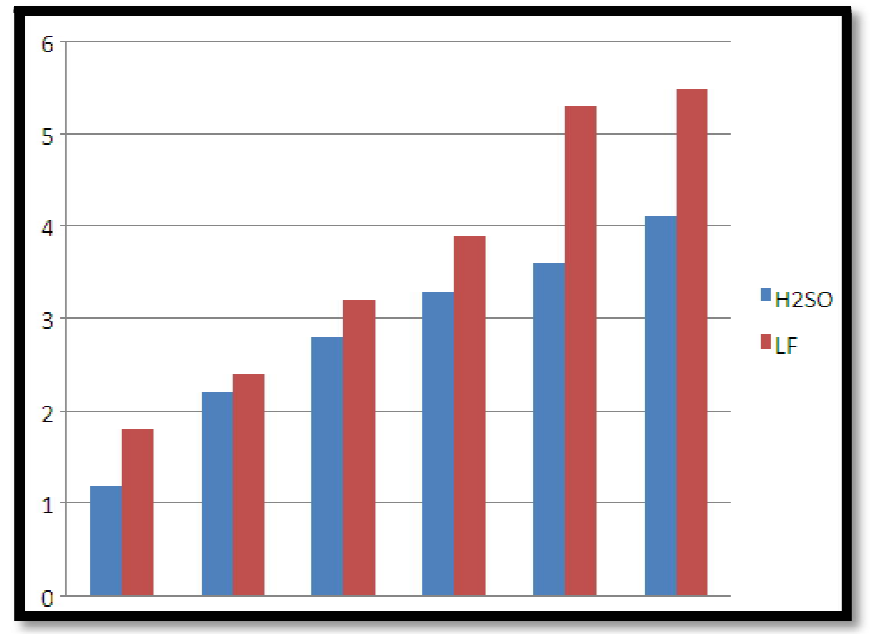

Figure 6: A Bar Chats Representing the Performance of H2SO4 and LFD at 1000 Rpm

\begin{tabular}{|c|c|c|c|c|c|c|}
\hline \multirow{2}{*}{$\begin{array}{c}\text { Vol. of } \\
\text { Demulsifier(ml) }\end{array}$} & \multicolumn{6}{|c|}{$\begin{array}{c}\text { Separated Water } \\
\text { Volume(ml) }\end{array}$} \\
\cline { 2 - 7 } & 2 min. & 4 min. & 6 min. & 8 min. & 10 min. & 12 min. \\
\hline 0.2 & 0.5 & 0.8 & 0.9 & 1.0 & 1.2 & 1.3 \\
\hline 0.4 & 1.0 & 1.1 & 1.4 & 1.6 & 1.7 & 2.3 \\
\hline 0.6 & 2.3 & 2.5 & 2.6 & 2.8 & 2.9 & 2.9 \\
\hline 0.8 & 2.9 & 3.0 & 3.3 & 3.3 & 3.4 & 3.4 \\
\hline 1.0 & 3.1 & 3.2 & 3.4 & 3.5 & 3.6 & 3.7 \\
\hline 1.2 & 4.2 & 4.5 & 4.9 & 5.1 & 5.2 & 5.2 \\
\hline
\end{tabular}

Table 6: Emulsion Treatment with H2SO4 at 1500 Rpm and 85of

Percentage volume of water separated using $\mathrm{H}_{2} \mathrm{SO}_{4}$ at $1500 \mathrm{rpm}$

$=$ Vol. water separated

Total vol. of water in the emulsion $\times \underline{100}$

$=\underline{5.2} \times \underline{100}$

$7.5 \quad 1=69.33 \%$

\begin{tabular}{|c|c|c|c|c|c|c|}
\hline \multirow{2}{*}{$\begin{array}{c}\text { Vol. of } \\
\text { Demulsifier(Ml) }\end{array}$} & \multicolumn{5}{|c|}{ Separated Water Volume(Ml) } \\
\cline { 2 - 7 } & $2 \mathrm{~min}$. & $4 \mathrm{~min}$. & $6 \mathrm{~min}$. & $8 \mathrm{~min}$. & $10 \mathrm{~min}$. & $12 \mathrm{~min}$. \\
\hline 0.2 & 0.8 & 1.2 & 1.5 & 1.8 & 2.0 & 2.3 \\
\hline 0.4 & 1.5 & 1.9 & 2.5 & 3.0 & 3.3 & 3.8 \\
\hline 0.6 & 3.0 & 3.8 & 4.0 & 4.2 & 4.3 & 4.3 \\
\hline 0.8 & 3.9 & 4.2 & 4.5 & 4.6 & 4.6 & 4.7 \\
\hline 1.0 & 4.5 & 4.9 & 5.0 & 5.2 & 5.5 & 6.9 \\
\hline 1.2 & 5.1 & 5.3 & 5.8 & 5.9 & 6.4 & 7.0 \\
\hline
\end{tabular}

Table 7: Emulsion Treatment with LFD at $1500 \mathrm{Rpm}$ and 85०

Percentage volume of water separated using LFD at 1500 RPM.

$=$ Vol. water separated $\quad x \quad 100$

Total vol. of water in the emulsion 1

$\underline{7.0} \times \underline{100}$

$$
\frac{7.0}{7.5} \times \frac{100}{1}=93.33 \%
$$




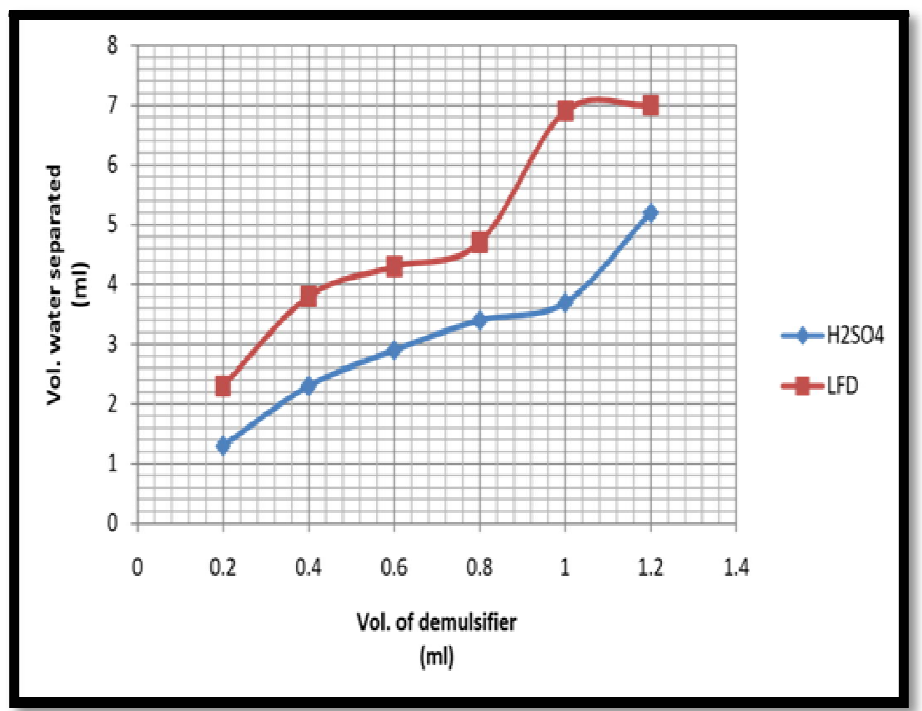

Figure 7: Vol. of Separated Water Vs Vol.

Demulsifier(Ml) @ 1500 Rpm and 85॰

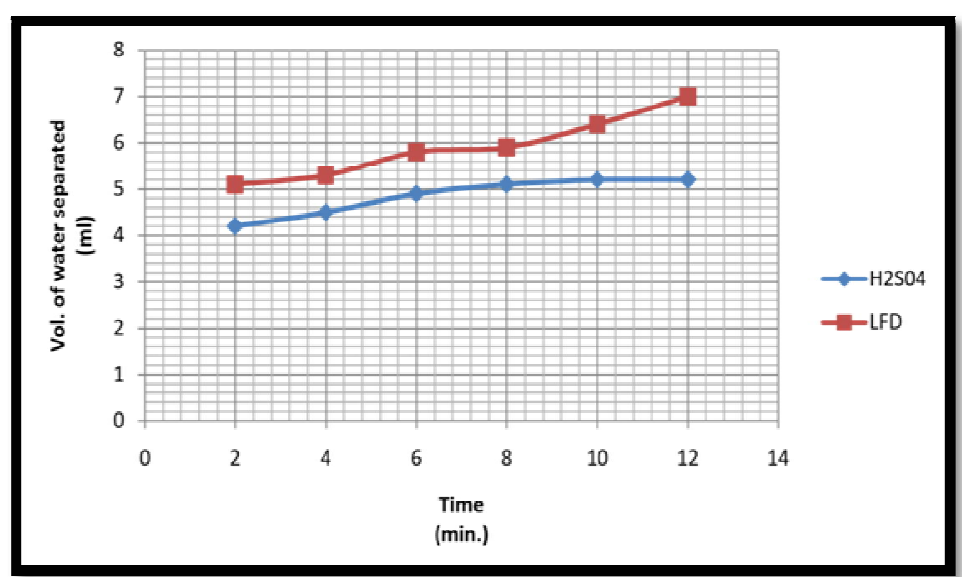

Figure 8: Volume of Water Separated (Ml) Vs

Time (Min) @1500 Rpm and 85of

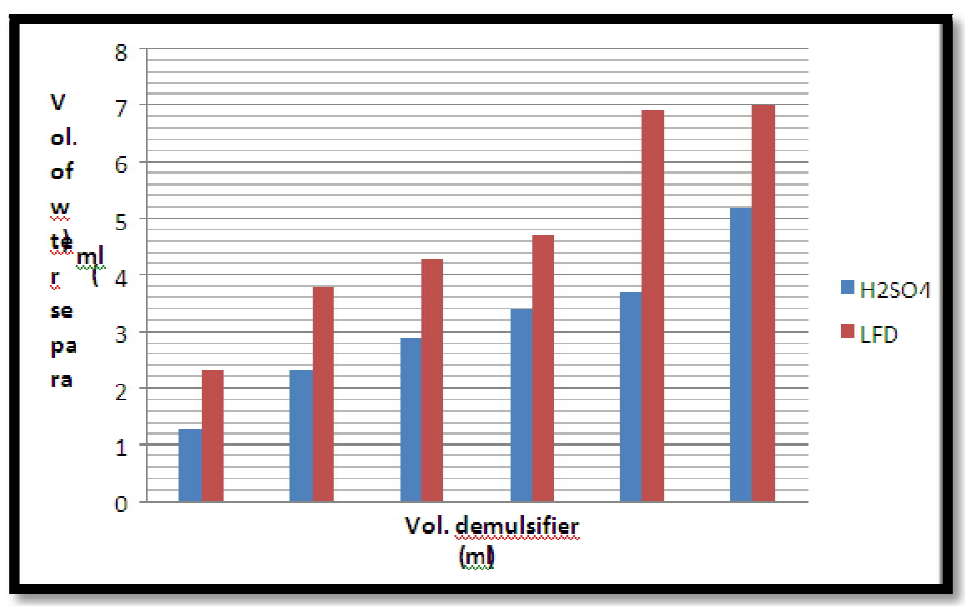

Figure 9: A Bar Chart Representing the Performance of H2so4 and Lfd @1500 Rpm and 85of 


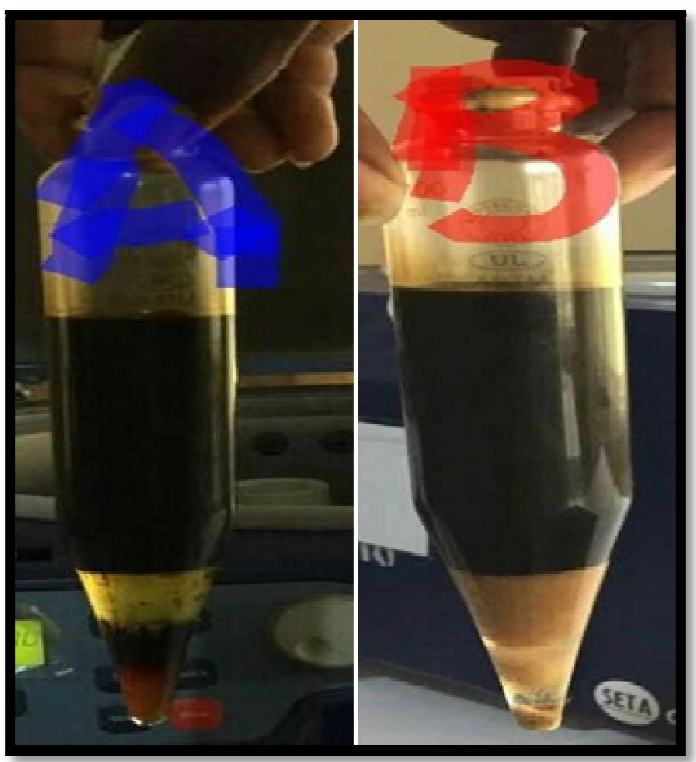

Figure 10: The Schematic Representation of Resolved Emulsion

From Table 3 the experiment was done without a Demulsifier and there was no separated water from the emulsion which shows that no amount of agitation will break an emulsion without a Demulsifier. At a speed of $1000 \mathrm{rpm}$ at $85 \mathrm{oF}$ better results were obtained using LFD as shown in Tables 3.3 and Fig 3.3. Percentage of water separated was $73.33 \%$. At a speed of $1500 \mathrm{rpm}$ at $85 \mathrm{oF}$ as displayed in Table 7 and Fig 3.5. The amount of water in percentage separated was $93.33 \%$. All the samples were agitated with centrifuge for 12 minutes in each case. This shows that locally formulated demulsifiers (LFD) were more effective in emulsion breaking than imported sulphuric acid ( H2SO4)

\section{Conclusion}

In oil field and water treatment operations, producer of crude oil is interested in three parts of demulsification. Rate or speed at which the detachment occurs, quantity of water remaining in the crude oil after separation, and quality of the detached water for disposal. In this work, a demulsifier was formulated from locally sourced material (LFD). It was tested on a crude oil emulsion sample from the Niger Delta's field in Nigeria at a temperature of 85oF in comparison with a commercially available imported demulsifier (sulfuric acid). The separated water percentage by volume using the locally formulated demulsifier (LFD) was 93.33\%, while the separated water using foreign demulsifier (Sulfuric acid) was $69.33 \%$. The LFD also had a faster rate of separation and a better quality of water separated than the commercially available imported demulsifer (sulfuric acid). The results clearly demonstrated that cheap local chemicals with demulsification properties can be used to successfully break emulsions and enhance the quality of produced crude oil. The locally formulated demulsifier (LFD) is not corrosive and does not contain organic chloride, bromides, or lead, hence will not cause any refining problems

\section{References}

i. Abdullah, S.Z.B. (2009). Separation of Crude Oil Emulsion via Ultrasonic Technology. Thesis submitted to the Faculty of Chemical and Natural Resources Engineering. in Partial Fulfillment of the Requirement for the Degree of Bachelor Engineering in Chemical Engineering Faculty of Chemical \& Natural Resources Engineering, University of Malaysia Pahang.

ii. Abdulrahaman, H.N., Rosli M. Y. and Zulkifly J. (2007). Chemical Demulsification of water-in-crude oil emulsions. Journal of Applied Sciences, 7(2), 10-21.

iii. Ahmad, S.B.O. (2009). Study on Emulsion Stability and Chemical Demulsification Characteristics. A thesis submitted in fulfillment of the requirements for the award of the degree of Bachelor of Chemical Engineering. Faculty of Chemical \& Natural Resources Engineering, University of Malaysia Pahang.

iv. Amir M. and Abdeni R. (2013). Using Demulsifiers for Phase Breaking of Water/ Oil Emulsion. A. Mosayebi, R Abedini/ Petroleum and Coal 55(1) 2630, Department of Petroleum Engineering, Mahallat Branch, Islamic Azad University, Mahallat, Iran. Available online at www.vurup.sk/ petroleumcoal.

v. Fingas, M. F., (2014). Water-in-Oil Emulsions: Formation and Prediction. Journal of Petroleum Science Research (JPSR), 3(1), 20-29.

vi. Hajivand, P. and Vaziri, A. (2015). Optimization of Demulsifier Formulation for Separation of Water from Crude Oil Emulsions. Brazilian Journal of Chemical Engineering, 32(1), 107-118.

vii. Isehunwa, S.O. \& Olanisebe, O. (2012). Interfacial tension of crude oil-brine systems in the Niger Delta. IJRRAS 10 (3), 10-12.

viii. Kokal, S. and Aramco, S. (2005). Crude Oil Emulsions: Everything You Wanted to Know but Were Afraid to Ask. SPE Distinguished Lecturer Series. 
ix. Manar, E. A. (2012). Factors Affecting the Stability of Crude Oil Emulsions. Petroleum Application Department Egyptian Petroleum Research Institute Egypt.

x. Manar, E.A. (2012). Crude Oil Emulsions - Composition Stability and Characterization. Petroleum Application Department. Egyptian Petroleum Research Institute Egypt.

xi. Martínez-Palou, Rafael, and Aburto, J. (2015). Ionic Liquids as Surfactants - Applications as Demulsifiers of Petroleum Emulsions. Available online at http:// www intechopen.com/ books/ ionic-liquids-current-state-oftheart.

xii. Ojinnaka, C.M., Ajienka, J.A. and Duru, R.U. (2016). Formulation of best-fit hydrophile/ lipophile balance-dielectric permittivity demulsifiers for treatment of crude oil emulsions. Egyptian Journal of Petroleum, 25(4), 565-574.

xiii. Pimeh, P.E. and Bello, K.O. (2016). A New Demulsifier Formulation for Crude Oil Emulsions Treatment. Petroleum Technology Development Journal, 6(2), 1-6.

xiv. Salam, K.K., Alade, A.O., Arinkoola, A.O. and Opawale, O. (2013). Improving the Demulsification Process of Heavy Crude Oil Emulsion through Blending with Diluent. A Journal of Petroleum Engineering, 7(9), 31-45.

xv. Tadros, T.F. (2013). Emulsion Formation, Stability, and Rheology. Wiley-VCH Verlag GmbH \& Co. KGaA. Published 2013 by Wiley-VCH Verlag GmbH \& Co. KGaA.

xvi. Udonne, J. D. (2012). Chemical treatment of emulsion problem in crude oil Production. Journal of Petroleum and Gas Engineering, 3(7), 135-141.

\section{Appendix}

\begin{tabular}{|l|l|}
\hline \multicolumn{2}{|c|}{ Nomenclature } \\
\hline BS \& W & Basic Sediments and Water \\
RPM & Revolution per minute \\
LFD & Locally formulated Demulsifier. \\
\hline
\end{tabular}

Table 8 